Canadian

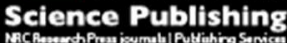

Biochemistry and Cell Biology Biochimie et biologie cellulaire

\title{
Osthole prevents cerebral ischemia/reperfusion injury via Notch signaling pathway
}

\begin{tabular}{|r|l|}
\hline Journal: & Biochemistry and Cell Biology \\
\hline Manuscript ID & bcb-2016-0233.R2 \\
\hline Manuscript Type: & Article \\
\hline Date Submitted by the Author: & O4-Feb-2017 \\
\hline Complete List of Authors: & $\begin{array}{l}\text { Guan, Junhong; Shengjing Hospital of China Medical University, } \\
\text { Department of Neurosurgery } \\
\text { Wei, Xiangtai; Shengjing Hospital of China Medical University } \\
\text { Qu, Shengtao; Shengjing Hospital of China Medical University } \\
\text { Lv, Tao; Shengjing Hospital of China Medical University } \\
\text { Fu, Qiang; Shengjing Hospital of China Medical University } \\
\text { Yuan, Ye; Shengjing Hospital of China Medical University }\end{array}$ \\
\hline Keyword: & \begin{tabular}{l} 
Osthole, stroke, ischemia/reperfusion, hippocampus neuron, Notch \\
\hline
\end{tabular} \\
\hline
\end{tabular}

\section{SCHOLARONE ${ }^{m}$}

Manuscripts 


\section{Osthole prevents cerebral ischemia/reperfusion injury via Notch}

\section{signaling pathway}

3

4

Junhong Guan*, Xiangtai Wei, Shengtao Qu, Tao Lv, Qiang Fu, Ye Yuan

Department of Neurosurgery, Shengjing Hospital of China Medical University, Shenyang 110004,

6 People's Republic of China

$7 \quad *$ Corresponding author: Dr. Junhong Guan, Department of Neurosurgery, Shengjing Hospital of

8 China Medical University, 36 Sanhao Street, Shenyang 110004, People's Republic of China

$9 \quad$ Tel: 024-96615-36211

10

E-mail: guanjunhong1962@sina.com 


\section{Abstract}

Stroke is a common cerebrovascular disease in aged population with the second leading mortality and the leading cause of permanent disability, and the ischemic stroke is the primary form. Osthole is a coumarin derivative extracted from the fruits of Cnidium monnieri (Linn.) Cuss. In this study, we established the rat middle cerebral artery occlusion/reperfusion (MCAO/R) model in vivo, and found that MCAO/R caused cerebral infarction, hippocampus neuronal injury and apoptosis, and also activated the Notch 1 signaling pathway. However, the treatment of Osthole further enhanced the activity of Notch 1 signaling, and reduced the cerebral infarction, hippocampus neuronal injury and apoptosis induced by MCAO/R in a dose-dependent manner. The same results were observed in primary neuronal oxygen glucose deficiency/reperfusion (OGD/R) model in vitro, and also the effect of Osthole could be blocked by a inhibitor of Notch 1 signaling, DAPT. Therefore, we demonstrated that Osthole injection prevented rat ischemia/reperfusion injury via activating Notch 1 signaling pathway in vivo and in vitro in a dose-dependent manner, which may be of great significant for clinical treatment of ischemic stroke.

Keywords: Osthole; stroke; ischemia/reperfusion; hippocampus neuron; Notch 


\section{Introduction}

The World Health Organization (WHO) reported in 2014 that stroke was the second leading cause of mortality with 6.7 million deaths each year around the world (WHO 2014). Stroke is also a leading cause of permanent disability (Kim et al. 2016). Stroke is categorized into two major subtypes, ischemic stroke and hemorrhagic stroke, wherein the former accounts for $80-85 \%$ (Kim et al. 2016). Ischemic stroke is caused by the formation of a blood clot in a cerebral artery, which is associated with hypertension, hyperlipidemia, diabetes mellitus and arteriosclerosis (Rosamond et al. 2007). Continuous ischemia leads to lesions and necrosis of brain tissue, and recovery of blood supply can rescue the reversible damage in brain tissue caused by ischemia (Schaller and Graf 2004). However, ischemia/reperfusion may result in additional injuries. Pathogenic edema and intracranial hemorrhage occurred in some ischemic stroke patients after thrombolysis. In several animal ischemic stroke models, reperfusion after ischemia for a long time would result in more serious cerebral infarction than permanent vascular ligation (Aronowski et al. 1997; Yang and Betz 1994). The mechanism of ischemia/reperfusion has not been completely illuminated, but it has been associated with reactive oxygen species (ROS), inflammation, mitochondria dysfunction, $\mathrm{Ca}^{2+}$ overload, apoptosis and autophagy.

Osthole (7-methoxy-8-isopentenoxy-coumarin) is a coumarin derivative extracted from a Chinese herbal medicine, the fruits of Cnidium monnieri (Linn.) Cuss. Since its anti-oxidant, anti-inflammation and anti-tumor potiential, Osthole has been used for gynecopathy and andrology diseases for many years. Recently, it has been reported that Osthole can ameliorate brain damage of mice and rats after ischemia via different signaling pathways (Chao et al. 2010;

Chen et al. 2015; Li et al. 2012; Li et al. 2016; Mao et al. 2011). 
51

52

53

In this study, we established rat middle cerebral artery occlusion/reperfusion (MCAO/R) model, and probed whether Osthole intervene the ischemia/reperfusion damage and the underlying mechanism.

\section{Methods}

\section{Ethical statement}

Animals in our study were taken care of according to Guide for the Care and Use of Laboratory Animals (1996, published by National Academy Press, 2101 Constitution Ave. NW, Washington, DC 20055, USA) and Laboratory Animal Care and Use Standard of China Medical University. Animal treatment and experimental procedures were approved by the Ethics Committee of China Medical University.

\section{Animal model}

Healthy adult male SD rats (250-280g) (Vital River, Beijing, China) were randomly divided into 5 groups: Sham, MCAO/R, MCAO/R+Ost(25), MCAO/R+Ost(50) and MCAO/R+Ost(100) ( $n=6$ per group). The rats in $M C A O / R+O s t(25), M C A O / R+O s t(50)$ and $M C A O / R+O s t(100)$ groups were intraperitoneally injected with Osthole (Aladdin, Shanghai, China) of $25 \mathrm{mg} / \mathrm{kg}, 50 \mathrm{mg} / \mathrm{kg}$ and 100 $\mathrm{mg} / \mathrm{kg}$, and the rats in Sham and MCAO/R groups were injected with the same volume of $10 \%$ Tween 80 as the control. 30 min later, all rats were anesthetized with $10 \%$ chloral hydrate $(3.5$ $\mathrm{mg} / \mathrm{kg}$ ), and ischemia for $120 \mathrm{~min}$ by Suture Embolization Method according to previous description (Jiang et al. 2016). The rats in Sham group were treated with the same operation but without ischemia. Then the reperfusion was induced, and rats in MCAO/R+Ost(25), $\mathrm{MCAO} / \mathrm{R}+\mathrm{Ost}(50)$ and $\mathrm{MCAO} / \mathrm{R}+\mathrm{Ost}(100)$ groups were intraperitoneally injected with Osthole of $25 \mathrm{mg} / \mathrm{kg}, 50 \mathrm{mg} / \mathrm{kg}$ and $100 \mathrm{mg} / \mathrm{kg}$ again, with the rats in Sham and MCAO/R groups injected 
with the same volume of $10 \%$ Tween 80 . After reperfusion for $24 \mathrm{~h}$, all rats were sacrificed with $10 \%$ chloral hydrate $(5 \mathrm{mg} / \mathrm{kg}$ ) through intreperitoneal injection, and the brain tissues were collected for the subsequent experiments.

\section{TTC staining}

The fresh brain tissues were frozen at $-20{ }^{\circ} \mathrm{C}$ for $30 \mathrm{~min}$ and cut into sections of $2 \mathrm{~mm}$. The sections were stained in $1 \%$ 2,3,5-tripheyl tetrazolium chloride (TTC) at $37{ }^{\circ} \mathrm{C}$ for $15 \mathrm{~min}$. During the staining process, the sections were turned over continually to be stained evenly. Thereafter, the percentage of infarction area was measured with ipp6.0. Five samples were estimated in each group, and the average value was calculated.

\section{Nissl staining}

The hippocampus tissue was fixed with $10 \%$ formaldehyde (Sinopharm) overnight, washed with water for $4 h$, and dehydrated with ethanol of $70 \%$ for $2 h, 80 \%$ for $2 h, 90 \%$ for $2 h$ and $100 \%$ for $1 \mathrm{~h}$ twice. After permeated with xylene (Sinopharm) for $30 \mathrm{~min}$, the tissue was embedded with paraffin at $60{ }^{\circ} \mathrm{C}$ and cut into sections of $5 \mu \mathrm{m}$. The sections were dried at $60{ }^{\circ} \mathrm{C}$, dewaxed with xylene for 15 min twice, and rehydrated with ethanol of different concentrations (100\% for 5 $\min$ twice, $95 \%$ for $2 \mathrm{~min}, 85 \%$ for $2 \mathrm{~min}$ and $75 \%$ for $2 \mathrm{~min}$ ) and water for $3 \mathrm{~min}$. Subsequently, the sections were stained with $0.5 \%$ cresol purple (Sinopharm) at temperature room for $10 \mathrm{~min}$, soaked with $0.25 \%$ acetic acid/ethanol (Kermel, Tianjin, China) for several seconds, and dehydrated with anhydrous ethanol (Sinopharm) for 5 min twice and xylene for 10 min twice. After wiping off residual liquid, the sections were mounted with half one drop of gum, and photographed with a microscope (Olympus, Tokoyo, Japan) at 200x magnification.

\section{TUNEL assay}


The hippocampus tissue was fixed with $10 \%$ formaldehyde (Sinopharm) at temperature overnight and washed with flow water for $4 \mathrm{~h}$. Subsequently, the tissue was dehydrated with ethanol of different concentrations (70\% for $2 \mathrm{~h}, 80 \%$ overnight, $90 \%$ for $2 \mathrm{~h}$ and $100 \%$ for $1 \mathrm{~h}$ twice), permeated with xylene (Sinopharm) for $30 \mathrm{~min}$, embedded with paraffin at $60{ }^{\circ} \mathrm{C}$ and cut into sections of $5 \mu \mathrm{m}$. The sections were dried on glass slides at $60{ }^{\circ} \mathrm{C}$, dewaxed with xylene for $15 \mathrm{~min}$ twice, hydrated with ethanol of $100 \%$ for 5 min twice, $95 \%$ for $2 \mathrm{~min}, 85 \%$ for $2 \mathrm{~min}$, $75 \%$ for $2 \mathrm{~min}$ and water for $2 \mathrm{~min}$. Then the sections were permeated with $0.1 \%$ Triton X-100 (Beyotime, Haimen, Jiangsu, China) for 8 min, blocked with $3 \% \mathrm{H}_{2} \mathrm{O}_{2}$ (Sinopharm) for 10 min and incubated with TUNEL reagent (Roche, Basel, Switzerland) at $37{ }^{\circ} \mathrm{C}$ in dark for $60 \mathrm{~min}$. After rinsing with PBS, the sections were incubated with Converter-POD (Roche) at $37{ }^{\circ} \mathrm{C}$ for $30 \mathrm{~min}$, reacted with DAB reagent (Solarbio, Beijing, China), stained with hematoxylin (Solarbio) for 3 min, soaked in $1 \%$ hydrochloric acid/ethanol for $3 \mathrm{~s}$, and washed with flow water for 20 min. Thereafter, the sections were dehydrated with ethanol of different concentrations ( $75 \%$ for $2 \mathrm{~min}$, $85 \%$ for $2 \mathrm{~min}, 95 \%$ for $2 \mathrm{~min}$ and 100\% for $10 \mathrm{~min}$ twice) and xylene for $10 \mathrm{~min}$ twice, and mounted with half one drop of gum. Finally, the sections were photographed with a microscope (Olympus) at $400 \times$ magnification.

\section{Immunofluorescence (IF) assay}

The hippocampus tissues were made into paraffin according to the previous description. The sections were dried at $60{ }^{\circ} \mathrm{C}$, dewaxed with xylene for 15 min twice, and hydrated with ethanol of $95 \%$ for $1 \mathrm{~min}, 85 \%$ for $1 \mathrm{~min}$ and 75\% for $1 \mathrm{~min}$. After washing with PBS for 5 min 3 times, the sections were repaired with antigen retrieval buffer for $10 \mathrm{~min}$, and washed with PBS for $5 \mathrm{~min}$ twice. After blocking with goat serum at room temperature for $30 \mathrm{~min}$, the sections were 
117

118

incubated with antibodies of mouse anti-NeuN (1:200) (Abcam, Cambridge, UK) and rabbit anti-cleaved caspase-3 (1:200) (CST, Boston, MA, USA) together at $4{ }^{\circ} \mathrm{C}$ overnight. After washing with PBS for 5 min 3 times, the sections were incubated with goat anti-mouse IgG labeled with Cy3 (1:200) (Beyotime) and goat anti-rabbit IgG labeled with FITC (1:200) (Beyotime) at room temperature for $90 \mathrm{~min}$ in dark. After washing with PBS for 5 min 3 times, the sections were counter-stained with DAPI, mounted in the presence of Antifade Mounting Medium (Solarbio), and photographed with a fluorescence microscope (Olympus) at 400x magnification.

\section{Western blot}

The total protein was extracted from hippocampus tissues or primary neurons by RIPA lysis buffer (Beyotime). After degenerating via boiling, the protein sample was separated by SDS-PAGE and transferred onto PVDF membrane (Millipore, Boston, MA, USA). After blocking with skim milk (YILI, Hohhot, Inner Mongolia, China) at room temperature for $1 \mathrm{~h}$, the PVDF membrane was incubated with the following antibodies at $4{ }^{\circ} \mathrm{C}$ overnight: rabbit anti-cleaved caspase-3 (Abcam, Cambrige, UK) (1:1000), rabbit anti-Bax (Boster) (1:400), rabbit anti-Bcl-2 (Boster, Wuhan, Hubei, China) (1:400), rabbit anti-Notch 1 (Abcam) (1:500), rabbit anti-NICD1 (Sangon, Shanghai, China) (1:500), and rabbit anti-Hes 1 (Boster) (1:400). After washing with PBS, the PVDF membrane was incubated with goat anti-rabbit IgG-HRP (Beyotime) (1:5000) at $37{ }^{\circ} \mathrm{C}$ for $45 \mathrm{~min}$, and explored with ECL reagent (7 sea, Shanghai, China). After removing the antibodies with stripping buffer (Beyotime), the PVDF membrane was incubated with mouse anti- $\beta$-actin (Santa Cruz, CA, USA) (1:1000) and goat anti-mouse IgG-HRP (Beyotime) (1:5000) to detect the internal control, $\beta$-actin.

\section{Primary neurons isolation and culture}

The hippocampus tissue isolated from neonatal SD rats was washed with PBS twice and cut 
139

140

141

142

143

144

145

into pieces of $1-3 \mathrm{~mm}^{3}$. Subsequently, the pieces were digested with trypsin at $37{ }^{\circ} \mathrm{C}$ for 30 min and centrifuged at $2500 \mathrm{rpm}$ for $10 \mathrm{~min}$. After removing the supernatant, the precipitation was resuspended with PBS, and centrifuged at $2500 \mathrm{rpm}$ for $10 \mathrm{~min}$. After removing the supernatant, the precipitation was resuspended with Neurbasal medium (Gibco BRL, Gaithersburg, MD, USA), and filtered with 200-mesh sieve to remove the tissue masses. The filtrate was centrifuged at $1000 \mathrm{rpm}$ for $10 \mathrm{~min}$, the cells in the precipitation was collected, washed with PBS twice, seeded into plates and cultured with Neurbasal medium added with $2 \% \mathrm{~B} 27$ (Gibco) at $37{ }^{\circ} \mathrm{C}$ in $5 \% \mathrm{CO}_{2}$. Establishment of the oxygen glucose deficiency/reperfusion (OGD/R) model: the neurons were cultured with Neurbasal medium (no glucose) in incubator with $5 \% \mathrm{CO}_{2}$ and $95 \% \mathrm{~N}_{2}$ for $2 \mathrm{~h}$, and then re-cultured in normal condition.

\section{MTT assay}

The neurons were seeded into 96 -well plates at a density of $4 \times 10^{3}$ per pore and cultured at $37{ }^{\circ} \mathrm{C}$ with $5 \% \quad \mathrm{CO}_{2} .22 \mathrm{~h}$ later, the neurons were treated with Osthole of different concentrations for $2 \mathrm{~h}$. Subsequently, the neurons were treated with OGD/R according to the previous description. After treating with $\mathrm{OGD} / \mathrm{R}$, the cells were added with MTT $(0.2 \mathrm{mg} / \mathrm{ml})$

(Sigma, St. Louis, MO, USA) to incubate for $4 \mathrm{~h}$ and centrifuged at $1000 \mathrm{rpm}$ for $10 \mathrm{~min}$. The supernatant was discarded, and $200 \mu$ of dimethyl sulfoxide (DMSO) (Sigma) was added to dissolve the formazan crystal. Subsequently, the optical density of the solution was detected with a microplate reader (BioTek, VT, USA) at $490 \mathrm{~nm}$.

\section{Lactic dehydrogenase (LDH) assay}

The LDH content in the cell culture supernatant was detected with LDH assay kit (Jiancheng, Nanjing, Jiangsu, China) according to the manufacturer's protocol. 
161

162

164

165

166

167

168

169

170

171

172

173

174

175

176

177

178

\section{Flow cytometry}

The neurons were seeded into plates and cultured with Neurbasal medium supplemented with 2\% B27. After culturing for $22 \mathrm{~h}$, the neurons were treated with Osthole of different concentrations for $2 \mathrm{~h}$, and treated with OGD/R. Thereafter, the neurons were collected, centrifuged at $800 \mathrm{rpm}$ for $5 \mathrm{~min}$. After removing the supernatant, the cells were washed with PBS and centrifuged at $800 \mathrm{rpm}$ for $5 \mathrm{~min}$ twice. Then the cells were treated with Annexin V-FITC/PI Apoptosis Detection Kit (KeyGEN, Nanjing, Jiangsu, China) according to the manufacturer's instruction, and detected with flow cytometry (BD, Franklin Lakers, NJ, USA).

\section{Statistical analysis}

The data in this study were presented as mean \pm standard deviation (SD), and analyzed by one-way ANOVA test. The data of animal tissues were collected from six individual experiments, and cell data were obtained from three individual experiments. It was considered statistically significant when $\mathrm{P}<0.05$. ( ${ }^{*} P<0.05, * * P<0.01, * * * P<0.001$, ns: no significance)

\section{Results}

\section{Osthole reduced ischemia-post cerebral infarction and hippocampus neuronal apoptosis}

The rat MCAO/R model was established by Suture Embolization Method and some rats were treated with Osthole of $25 \mathrm{mg} / \mathrm{kg}, 50 \mathrm{mg} / \mathrm{kg}$ and $100 \mathrm{mg} / \mathrm{kg}$. Subsequently, TTC staining was used to detect the cerebral infarction of rats with different treatments. The results showed that MCAO/R led to an infarction of $39.4 \%$ in cerebra, and Osthole decreased the infarction by $3.3 \%$, $22.8 \%$ and $32.8 \%$ (Fig. $1 \mathrm{~A}$ and $\mathrm{B}$ ). Then Nissl staining results revealed that MCAO/R caused hippocampus neurons injury and the injury was mitigated by the treatment of Osthole (Fig. $1 \mathrm{C}$ and E). The similar results were observed in TUNEL assay, namely MCAO/R induced hippocampus 
183

184

185

186

187

neuronal apoptosis and Osthole alleviated the apoptosis (Fig. 1D and F). Thereafter, IF was performed to measure apoptosis by testing the apoptosis executor, cleaved caspase-3, and a neuronal marker, NeuN. As shown in Fig. 2, MCAO/R increased the level of cleaved caspase-3 and decreased the level of NeuN significantly, which were rescued by Osthole (Fig. 2A-C). Finally, we measured the expression levels of cleaved caspase-3, Bax and Bcl-2, by western blot. As shown in Fig. 2, after MCAO/R, cleaved caspase-3 was increased by 3.2-folds, Bax was elevated by 3.41-folds, and Bcl-2 was decreased by 70\% (Fig. 2D and E). However, the treatment of Osthole of $25 \mathrm{mg} / \mathrm{kg}, 50 \mathrm{mg} / \mathrm{kg}$ and $100 \mathrm{mg} / \mathrm{kg}$ descended cleaved caspase-3 by $41.6 \%, 50.8 \%$ and $54.8 \%$, reduced Bax by $27.6 \%, 37.7 \%$ and $52.2 \%$, and ascended Bcl-2 by 1.4 folds, 2.32 folds and 2.6 folds (Fig. 2D and E), respectively.

\section{Osthole enhanced the activity of Notch 1 signaling pathway in vivo}

To investigate the relationship among MCAO/R, Osthole and Notch 1, we detect the expression levels of Notch 1, Notch intracellular domain (NICD) 1 and a canonical target of Notch 1, Hes 1

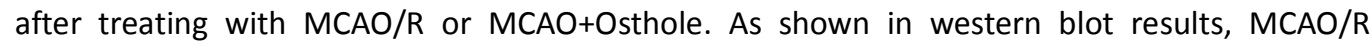
increased the expression of Notch 1 by 1.86 -folds, NICD by 1.34 -fold and Hes 1 by 1.6 -fold (Fig. 2F and G). Moreover, after treatment with Osthole, the level of Notch 1 further increased by 1.06-fold, 1.17-fold and 1.45-fold, the level of NICD 1 further increased by 1.1-fold, 1.26-fold and 1.65-fold, and the level of Hes 1 further increased by 1.03-fold, 1.13-fold and 1.5-fold (Fig. 2F and

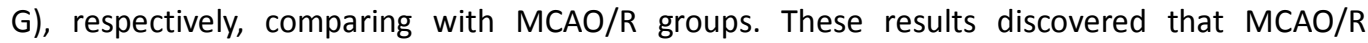
activated the Notch 1 signaling pathway and the Osthole strengthened the activity in a dose-dependent manner.

\section{Osthole increased the viability of primary neuron}


The above results proved that MCAO/R induced cerebral infarction and neuronal injury, and activated Notch 1 signaling pathway in vivo, next we explored whether Osthole reduced neuronal injury via Notch 1 signaling pathway in vitro. The primary neurons were isolated from hippocampus tissues of fetal rats, and cultured in glucose-free medium without $\mathrm{O}_{2}$ to simulate the ischemia/reperfusion in vitro. After pre-treating with Osthole of $10 \mu \mathrm{M}, 50 \mu \mathrm{M}$ and $100 \mu \mathrm{M}$, the neurons were detected by MTT assay to measure the viability. The results showed that OGD/R decreased the neuronal viability by $43 \%$, and the pre-treatment of Osthole recovered the viability by $19 \%, 87 \%$ and $84 \%$ (Fig. $3 A$ ). Then a common cerebral damage marker, LDH, was measured. As exhibited in the results, OGD/R enhanced the LDH activity by 3.8-fold, but the Osthole of $10 \mu \mathrm{M}, 50 \mu \mathrm{M}$ and $100 \mu \mathrm{M}$ weakened the LDH activity by $96 \%, 65 \%$ and 59\% (Fig. 3B). To probe whether Oshole played a role via Notch 1 pathway, a $\gamma$-secretase inhibitor, $\mathrm{N}$-[N-(3,5-difluorophenacetyl)-L-alanyl]-S-phenylglycine t-butyl ester (DAPT) was used with $1 \mu \mathrm{M}$. Considering the effect of Osthole of different concentrations, $50 \mu \mathrm{M}$ was selected as the experimental concentration in the subsequently experiments. Then we detected the neuronal viability and found that DAPT inhibited the function of Osthole in some degree (Fig. 3C), and similar results were observed in LDH enzymatic activity assay results (Fig. 3D).

\section{Osthole inhibited the neuronal apoptosis induced by $O G D / R$ and the inhibition was reversed by}

\section{DAPT}

Next, we tested the apoptosis of primary neurons in vitro by flow cytometry. The results revealed that $\mathrm{OGD} / \mathrm{R}$ enhanced the apoptosis rate of primary neurons by $9.2 \%$, but the enhancement was inhibited $52 \%$ by Osthole, however, the inhibition was neutralized by DAPT partly (Fig. 4A and B). Then the expression levels of cleaved caspase-3, Bax and Bcl-2 in the 
227

primary neurons were detected by western blot. As similar to the results in vivo, after treating with $\mathrm{OGD} / \mathrm{R}$, the levels of cleaved caspase-3 and Bax were increased, and the level of Bcl-2 was decreased, which were recovered by Osthole. However, the recovery was relieved by DAPT partly (Fig. 4C and D), suggesting that Osthole inhibited the ischemia/reperfusion-induced apoptosis of neurons by activating Notch pathway.

\section{Osthole promoted the activity of Notch 1 signaling pathway in vitro}

To verify the hypothesis that Osthole alleviated the neuronal apoptosis induced by ischemia/reperfusion via Notch 1 signaling pathway, we detect the expression levels of Notch 1 , NICD 1 and Hes 1 in the primary neurons with different treatments. As expected, OGD/R increased the levels of Notch 1 by 1.6 -fold, NICD 1 by 1.9 -fold and Hes 1 by 1.5 -fold, and Osthole further promoted the expression of Notch 1 by 1.4-fold, NICD 1 by 1.3 -fold and Hes 1 by 1.5 -fold. However, the increase was suppressed by DAPT, an inhibitor of Notch pathway (Fig. 4E and F).

\section{Discussion}

Notch is a class of highly conserved cell surface receptors. There are four isomers of Notch in mammals, Notch 1-4. Notch is a transmembrane protein, including extracellular domain and intracellular domain. In the canonical Notch signaling pathway, when the extracellular domain binds to the ligand, the intracellular domain is hydrolyzed twice by tumor necrosis factor (TNF)- $\alpha$ inverting enzyme (TACE) and $\gamma$-secretase into the activated form, Notch intracellular domain (NICD). Subsequently, NICD translocates into the nuclear, and binds to the transcriptional factor CBF-1/suppressor of hairless/LAG-1 (CSL), to activate the expression of downstream genes (Crabtree et al. 2016). CSL is a transcriptional inhibitor when it exists alone, but induces the transcription of corresponding genes after combining with NICD (Kovall and Blacklow 2010). 
Notch 1 is highly expressed in neural stem cells, and regulates neural development and dendrite morphology (Ding et al. 2016). What's more, Notch 1 signaling pathway was activated after ischemia in vivo and in vitro, with a neural repair function. The activation of Notch 1 could promote the proliferation and differentiation of neural progenitor cells after ischemic stroke (Wang et al. 2009a). Increased activation of Notch 1 enhances neurogenesis in the subventricular zone (SVZ) of ischemic rat brain, and inhibiting Notch 1 signaling results in a reduction of proliferating cells SVZ cells in the SVZ (Wang et al. 2009b). It has also been reported that Notch signaling promotes macrophage activation and dendritic cells differentiation after stroke (Brifault et al. 2015; Shang et al. 2016). Notch signaling contributes to the maintenance of NF-KB activation, and enhances the inflammatory response (Cai et al. 2016; Zhang et al. 2015). In addition, Notch 1 activates microglia after focal cerebral ischemia, which is able to mediate the inflammation, clear the damaged neurons and repair the injury after ischemia (Wei et al. 2011). In addition, ischemic injury could be alleviated by several drugs via Notch 1 signaling (Chen et al. 2008; Liu et al. 2016; Yuan et al. 2015). In this study, we established rat MCAO/R model and found that MCAO/R caused cerebral infarction, neuronal injury and apoptosis, and activated and reduced the cerebral infarction, neuronal injury and apoptosis caused by MCAO/R in a dose-dependent manner. The same results were observed in primary neurons treating with OGD/R in vitro. 
271 2002; Rooman et al. 2006; Shang et al. 2016). On the other hand, since extracting from a

272 traditional Chinese medicine, Osthole has been used for many years with its anti-inflammation,

273 bacteriostat and anti-tumor functions. In this study, we demonstrated that Osthole ameliorated

274 decreased cerebral infarction, restrained neural apoptosis induced by ischemia/reperfusion in

275 vivo and in vitro via Notch signaling pathway. These findings may provide some research basic for

276 clinical treatment of ischemia stroke.

\section{Conclusion}

278 In this study, we established rat MCAO/R model and demonstrated that Osthole prevented

279 cerebral infarction, hippocampus neuronal injury and apoptosis induced by MCAO/R by activating

280 Notch 1 signaling pathway in a dose-dependent manner in vivo. Similar results were observed in

281 OGD/R-treated primary neurons in vitro. These findings may provide some research basic for

282 clinical therapy of ischemia/reperfusion. 
Acknowledgements

284 This study was supported grants from the Natural Science Foundation of Liaoning Province (No.

$2852015020504)$

286 Conflict of interest

287 There is no conflict of interest between authors of this article, neither between authors and

288 any other institutions or individuals. 


\section{References}

Aronowski, J., Strong, R., and Grotta, J.C. 1997. Reperfusion injury: demonstration of brain damage produced by reperfusion after transient focal ischemia in rats. J Cereb Blood Flow Metab 17(10): 1048-1056.

Brifault, C., Gras, M., Liot, D., May, V., Vaudry, D., and Wurtz, O. 2015. Delayed pituitary adenylate cyclase-activating polypeptide delivery after brain stroke improves functional recovery by inducing $\mathrm{m} 2$ microglia/macrophage polarization. Stroke 46(2): 520-528.

Cai, Z., Zhao, B., Deng, Y., Shangguan, S., Zhou, F., Zhou, W., Li, X., Li, Y., and Chen, G. 2016. Notch signaling in cerebrovascular diseases (Review). Mol Med Rep 14(4): 2883-2898.

Chao, X., Zhou, J., Chen, T., Liu, W., Dong, W., Qu, Y., Jiang, X., Ji, X., Zhen, H., and Fei, Z. 2010. Neuroprotective effect of osthole against acute ischemic stroke on middle cerebral ischemia occlusion in rats. Brain Res 1363: 206-211.

Chen, J., Zacharek, A., Li, A., Cui, X., Roberts, C., Lu, M., and Chopp, M. 2008. Atorvastatin promotes presenilin-1 expression and Notch1 activity and increases neural progenitor cell proliferation after stroke. Stroke 39(1): 220-226.

Chen, Z., Mao, X., Liu, A., Gao, X., Chen, X., Ye, M., Ye, J., Liu, P., Xu, S., Liu, J., He, W., Lian, Q., and Pi, R. 2015. Osthole, a natural coumarin improves cognitive impairments and BBB dysfunction after transient global brain ischemia in $\mathrm{C} 57 \mathrm{BL} / 6 \mathrm{~J}$ mice: involvement of Nrf2 pathway. Neurochem Res 40(1): 186-194.

Crabtree, J.S., Singleton, C.S., and Miele, L. 2016. Notch Signaling in Neuroendocrine Tumors. Front Oncol 6: 94.

Ding, X.F., Gao, X., Ding, X.C., Fan, M., and Chen, J. 2016. Postnatal dysregulation of Notch signal 
311

312

313

314

315

disrupts dendrite development of adult-born neurons in the hippocampus and contributes to memory impairment. Sci Rep 6: 25780.

Hitoshi, S., Alexson, T., Tropepe, V., Donoviel, D., Elia, A.J., Nye, J.S., Conlon, R.A., Mak, T.W., Bernstein, A., and van der Kooy, D. 2002. Notch pathway molecules are essential for the maintenance, but not the generation, of mammalian neural stem cells. Genes Dev 16(7): 846-858.

Jiang, D., Wang, Y., Zang, Y., Liu, X., Zhao, L., Wang, Q., Liu, C., Feng, W., Yin, X., and Fang, Y. 2016. Neuroprotective Effects of rhGLP-1 in Diabetic Rats with Cerebral Ischemia/Reperfusion Injury. Drug Dev Res 77(3): 124-133.

Kim, J., Fann, D.Y., Seet, R.C., Jo, D.G., Mattson, M.P., and Arumugam, T.V. 2016. Phytochemicals in Ischemic Stroke. Neuromolecular Med.

Kovall, R.A., and Blacklow, S.C. 2010. Mechanistic insights into Notch receptor signaling from structural and biochemical studies. Curr Top Dev Biol 92: 31-71.

Li, F., Gong, Q., Wang, L., and Shi, J. 2012. Osthole attenuates focal inflammatory reaction following permanent middle cerebral artery occlusion in rats. Biol Pharm Bull 35(10): 1686-1690.

Li, K., Ding, D., and Zhang, M. 2016. Neuroprotection of Osthole against Cerebral Ischemia/Reperfusion Injury through an Anti-apoptotic Pathway in Rats. Biol Pharm Bull 39(3): $336-342$.

Liu, S., Cao, Y., Qu, M., Zhang, Z., Feng, L., Ye, Z., Xiao, M., Hou, S.T., Zheng, R., and Han, Z. 2016. Curcumin protects against stroke and increases levels of Notch intracellular domain. Neurol Res 38(6): 553-559.

Mao, X., Yin, W., Liu, M., Ye, M., Liu, P., Liu, J., Lian, Q., Xu, S., and Pi, R. 2011. Osthole, a natural 
333

coumarin, improves neurobehavioral functions and reduces infarct volume and matrix metalloproteinase-9 activity after transient focal cerebral ischemia in rats. Brain Res 1385: $275-280$.

Rooman, I., De Medts, N., Baeyens, L., Lardon, J., De Breuck, S., Heimberg, H., and Bouwens, L. 2006. Expression of the Notch signaling pathway and effect on exocrine cell proliferation in adult rat pancreas. Am J Pathol 169(4): 1206-1214.

Rosamond, W., Flegal, K., Friday, G., Furie, K., Go, A., Greenlund, K., Haase, N., Ho, M., Howard, V., Kissela, B., Kittner, S., Lloyd-Jones, D., McDermott, M., Meigs, J., Moy, C., Nichol, G., O'Donnell, C.J., Roger, V., Rumsfeld, J., Sorlie, P., Steinberger, J., Thom, T., Wasserthiel-Smoller, S., Hong, Y., American Heart Association Statistics, C., and Stroke Statistics, S. 2007. Heart disease and stroke statistics--2007 update: a report from the American Heart Association Statistics Committee and Stroke Statistics Subcommittee. Circulation 115(5): e69-171.

Schaller, B., and Graf, R. 2004. Cerebral ischemia and reperfusion: the pathophysiologic concept as a basis for clinical therapy. J Cereb Blood Flow Metab 24(4): 351-371.

Shang, Y., Smith, S., and Hu, X. 2016. Role of Notch signaling in regulating innate immunity and inflammation in health and disease. Protein Cell 7(3): 159-174.

Wang, L., Chopp, M., Zhang, R.L., Zhang, L., Letourneau, Y., Feng, Y.F., Jiang, A., Morris, D.C., and Zhang, Z.G. 2009a. The Notch pathway mediates expansion of a progenitor pool and neuronal differentiation in adult neural progenitor cells after stroke. Neuroscience 158(4): 1356-1363.

Wang, X., Mao, X., Xie, L., Greenberg, D.A., and Jin, K. 2009b. Involvement of Notch1 signaling in neurogenesis in the subventricular zone of normal and ischemic rat brain in vivo. J Cereb Blood Flow Metab 29(10): 1644-1654. 
355

356

357

358

359

360

361

362

363

364

365

366

367

368

369

Wei, Z., Chigurupati, S., Arumugam, T.V., Jo, D.G., Li, H., and Chan, S.L. 2011. Notch activation enhances the microglia-mediated inflammatory response associated with focal cerebral ischemia.

Stroke 42(9): 2589-2594.

WHO. 2014 The top 10 causes of death. http://www.who.int/mediacentre/factsheets/fs310/en/.

Yang, G.Y., and Betz, A.L. 1994. Reperfusion-induced injury to the blood-brain barrier after middle cerebral artery occlusion in rats. Stroke 25(8): 1658-1664; discussion 1664-1655.

Yuan, Y., Rangarajan, P., Kan, E.M., Wu, Y., Wu, C., and Ling, E.A. 2015. Scutellarin regulates the Notch pathway and affects the migration and morphological transformation of activated microglia in experimentally induced cerebral ischemia in rats and in activated BV-2 microglia. J Neuroinflammation 12: 11.

Zhang, Y., He, K., Wang, F., Li, X., and Liu, D. 2015. Notch-1 signaling regulates astrocytic proliferation and activation after hypoxia exposure. Neurosci Lett 603: 12-18. 
370

371

372

373

374

375

376

377

\section{Figure legends}

Fig. 1 Osthole reduced the cerebral infarction and hippocampus neuronal injury induced by middle cerebral artery occlusion/reperfusion (MCAO/R)

(A) TTC staining was performed to detect cerebral infarction of rats suffering from MCAO/R or Osthole. The scale bar represented $4 \mathrm{~mm}$. (B) The cerebral infarction volume percentage of rats in each group. (C) Nissl staining was used to detect the hippocampus neuronal injury of rats in each group. The scale bar represented $100 \mu \mathrm{m}$. (D) TUNEL assay was carried out to test hippocampus neuronal apoptosis of rats in each group. The scale bar represented $50 \mu \mathrm{m}$. The representative image was taken at $600 \times$ magnification, and the TUNEL-positive cells were indicated with arrows.

(E) Quantification of Nissl staining of hippocampus neurons. (F) Quantification of TUNEL-positive cells. ( ${ }^{*} P<0.05, * * * P<0.001$, ns: no significance)

Fig. 2 Osthole alleviated MCAO/R-induced hippocampus neuronal apoptosis and enhanced the activity of Notch 1 signaling pathway in vivo

(A) Immunofluorescence (IF) was used to measure the neuronal apoptosis by testing cleaved caspase-3 and NeuN in hippocampus tissue of rats with different treatments. The scale bar represented $50 \mu \mathrm{m}$. (B) Quantification of NeuN fluorescence signal. (C) Quantification of cleaved caspase-3 fluorescence signal. (D) The expression levels of cleaved caspase-3, Bax and Bcl-2 in the hippocampus tissues were detected by western blot. (E) The western blot bands of cleaved caspase-3, Bax and Bcl-2 in the hippocampus tissues. (F) The expression level of Notch 1, Notch intracellular domain (NICD) 1 and Hes 1 in the hippocampus tissues was detected by western blot. (G) The western blot bands of Notch 1, Notch intracellular domain (NICD) 1 and Hes 1 in the hippocampus tissues. ( ${ }^{*} P<0.05,{ }^{*} P<0.01,{ }^{* * *} P<0.001$, ns: no significance) 
392

393

394

395

396

397

398

399

400

401

402

403

404

405

406

407

408

Fig. 3 Osthole increased the viability of primary neuron

(A) The MTT assay was performed to measure the viability of primary neurons treated with Osthole of $10 \mu \mathrm{M}, 50 \mu \mathrm{M}$ and $100 \mu \mathrm{M}$. (C) The viability of primary neuron suffering from different treatments was tested at $490 \mathrm{~nm}$. (B) The enzymatic activity of lactic dehydrogenase (LDH) in primary neurons culture supernatant treated with Osthole of $10 \mu \mathrm{M}, 50 \mu \mathrm{M}$ and $100 \mu \mathrm{M}$. (D) The

LDH activity in culture supernatant of primary neurons under different treatements was detected. $\left({ }^{*} P<0.01, * * * P<0.001\right.$, ns: no significance)

Fig. 4 Osthole inhibited OGD/R-induced neuronal apoptosis and promoted the activity of Notch

\section{1 signaling pathway in vitro}

(A) Flow cytometry was performed to measure primary neuronal apoptosis with different treatments. (B) Apoptosis rate of neurons in each group. (C) The expression levels of cleaved caspase-3, Bax and Bcl-2 in primary neurons were tested by western blot. (D) The western blot bands of cleaved caspase-3, Bax and Bcl-2 in primary neurons. (E) Western blot was used to measure the expression levels of Notch 1, NICD 1 and Hes 1 in primary neuron with different treatments. (F) The western blot bands of Notch 1, NICD 1 and Hes 1 in primary neuron with different treatments. $\left({ }^{*} P<0.05,{ }^{* *} P<0.01,{ }^{* * *} P<0.001\right)$ 

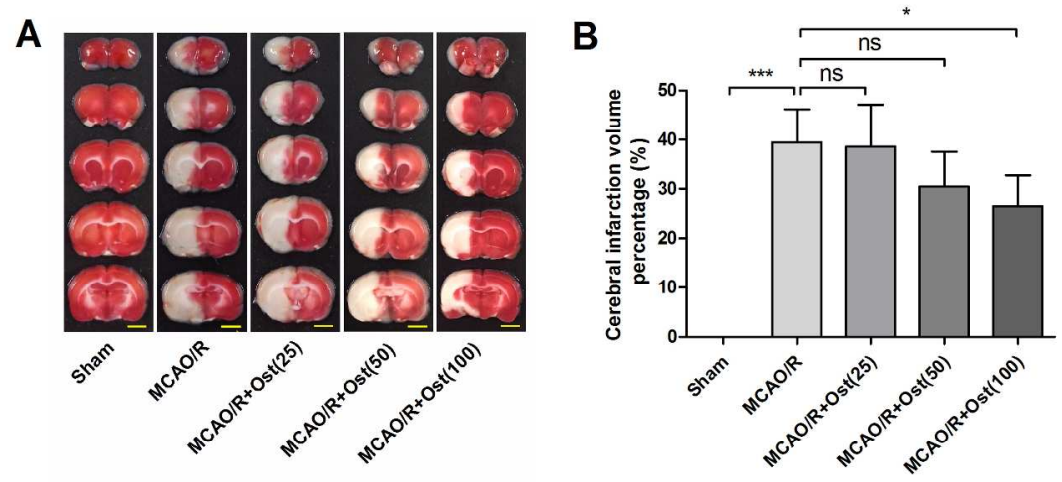

C

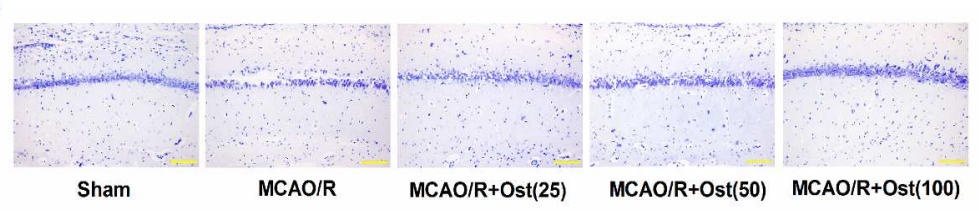

D

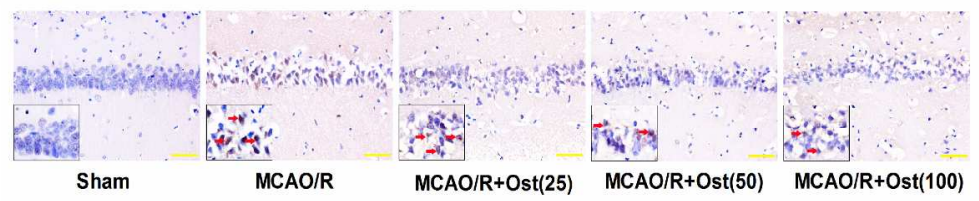

E

$\mathbf{F}$
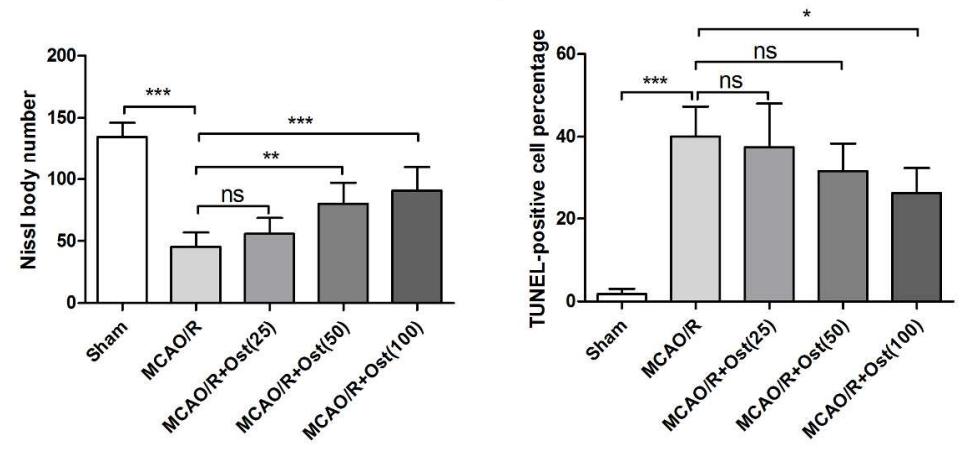

figure 1

$199 \times 285 \mathrm{~mm}(300 \times 300$ DPI $)$ 


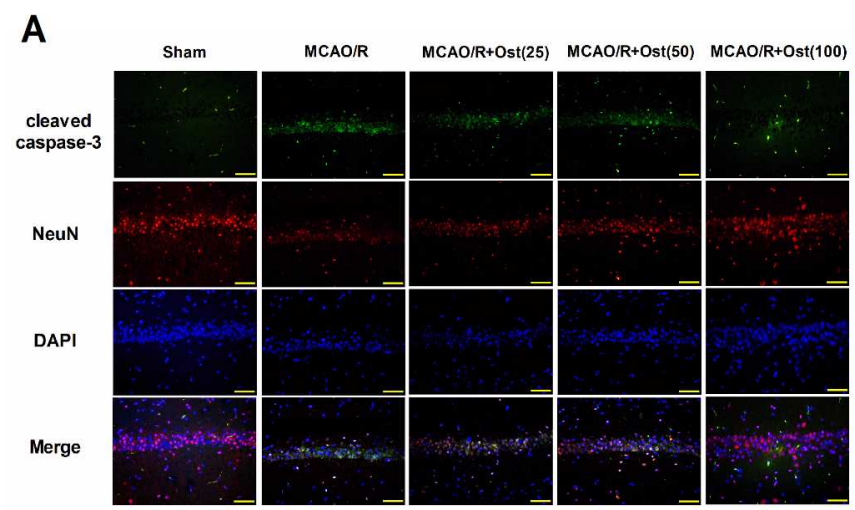

B

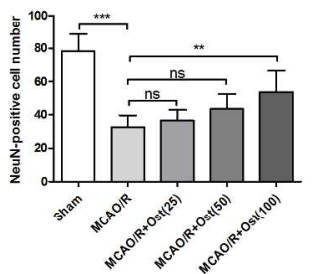

C

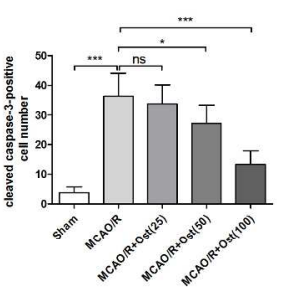

D

$\square$ Sham $\square$ MCAO/R $\square$ MCAO/R+OSt(25)

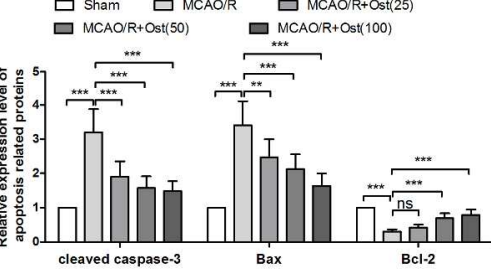

E

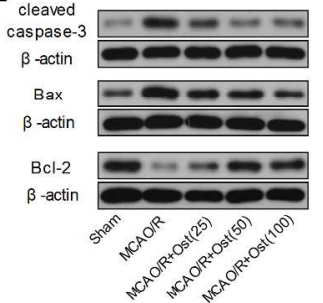

$\mathbf{F}$

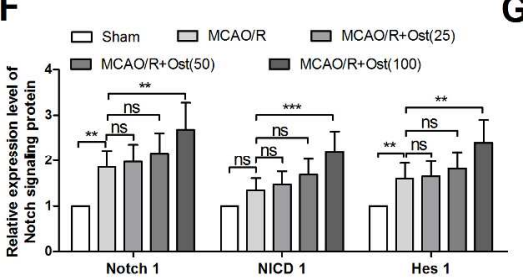

G

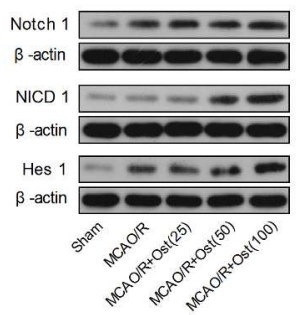

figure 2

$171 \times 295 \mathrm{~mm}(300 \times 300$ DPI $)$ 
A

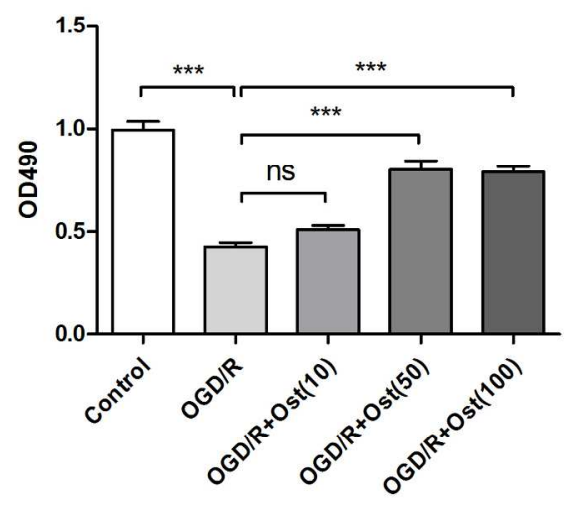

C

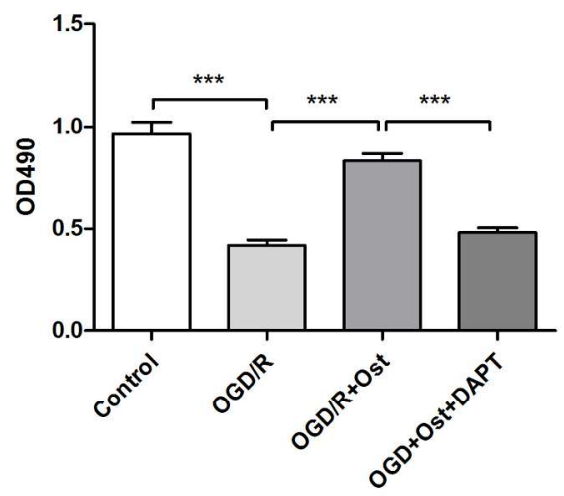

B

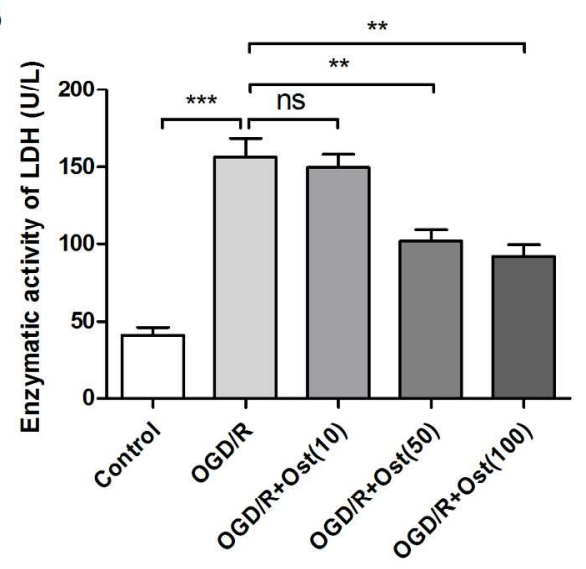

D

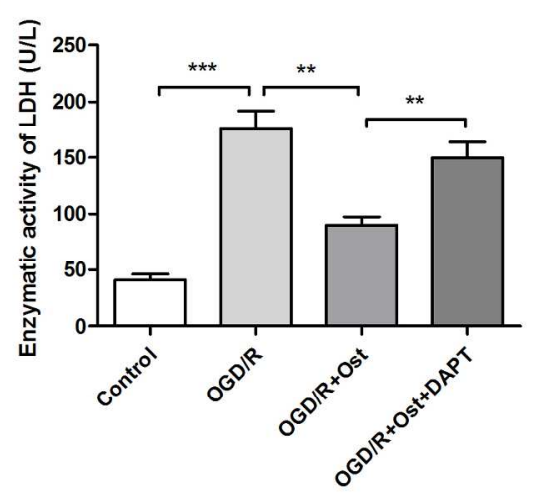

figure 3

$186 \times 174 \mathrm{~mm}(300 \times 300$ DPI $)$ 
A
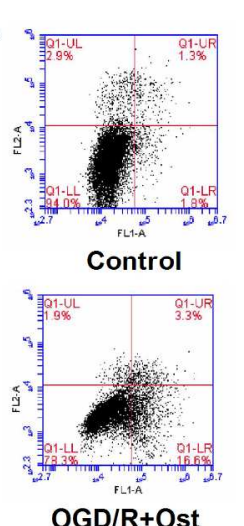

OGD/R+Ost
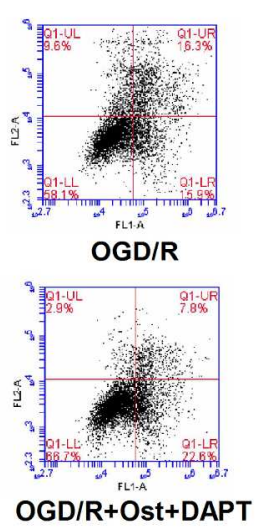

B

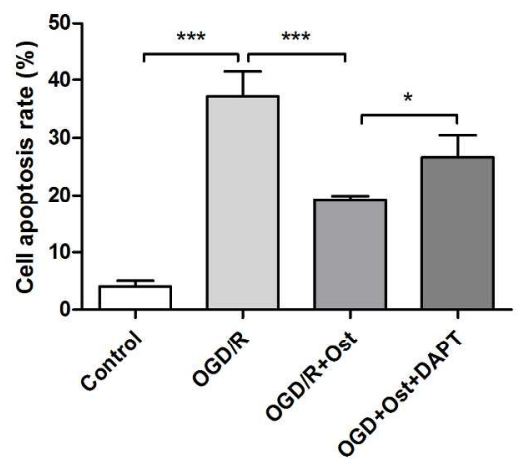

C

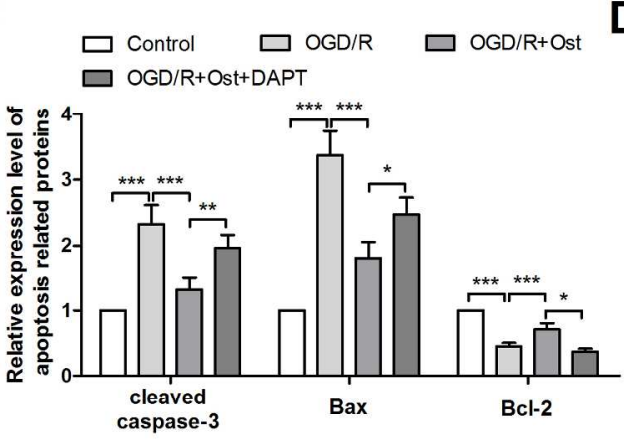

E

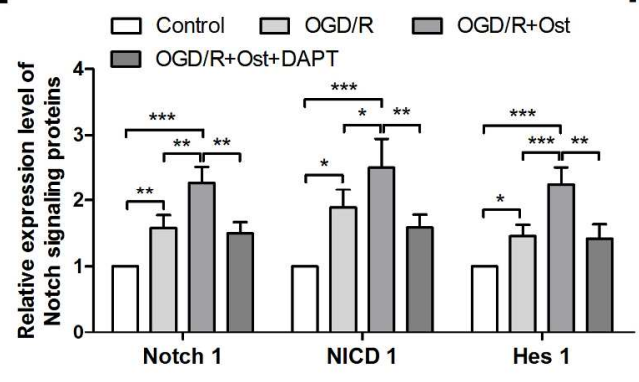

D cleaved caspase-3 $\beta$-actin

Bax $\beta$-actin Bcl-2 $\beta$-actin
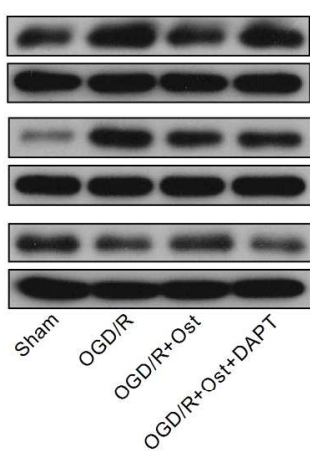

$\mathbf{F}$

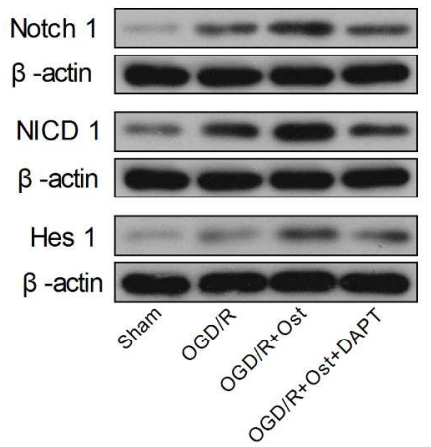

figure 4

$185 \times 247 \mathrm{~mm}(300 \times 300$ DPI $)$ 